\title{
Dynamical and structural insights into the smectic phase of rod-like particles
}

\author{
Eric Grelet ${ }^{1}$, M. Paul Lettinga ${ }^{2}$, Markus Bier ${ }^{3}$, René van Roij ${ }^{3}$, \\ and Paul van der Schoot ${ }^{4}$ \\ ${ }^{1}$ Centre de Recherche Paul Pascal, CNRS-Université Bordeaux 1, 115 Avenue \\ Schweitzer, 33600 Pessac, France \\ ${ }^{2}$ IFF, Institut Weiche Materie, Forschungszentrum Jülich, D-52425 Jülich, Germany \\ 3 Institute for Theoretical Physics, Utrecht University, Leuvenlaan 4, $3584 \mathrm{CE}$ \\ Utrecht, The Netherlands \\ 4 Department of Applied Physics, Eindhoven University of Technology, P.O. Box 513, \\ 5600 MB Eindhoven, The Netherlands \\ E-mail: grelet@crpp-bordeaux.cnrs.fr
}

\begin{abstract}
.
Self-diffusion in a model system of rod-like particles is studied in the smectic (or lamellar) phase. The experimental system is formed by a colloidal suspension of filamentous $f d$ virus particles, which allows the direct visualisation at the scale of the single particle of mass transport between the smectic layers. Self-diffusion takes place preferentially in the direction normal to the smectic layers and occurs in steps of one rod length, reminiscent of a hopping-type of transport. The probability density function is obtained experimentally at different times and is found to be in qualitative agreement with theoretical predictions based on a dynamical density functional theory.
\end{abstract}

PACS numbers: 61.30.-v, 82.70.Dd, 87.15.Vv

\section{Introduction}

The self-organisation into liquid-crystalline states is a field of intensive research, both theoretically [1] and experimentally [2,3]. Recently, the dynamics of such self-assembled anisotropic media has been investigated in particular by the determination of selfdiffusion coefficients in different kinds of mesophases [4]. These measurements have been performed with experimental techniques probing the samples collectively (ensemble averaged), such as in nuclear magnetic resonance (NMR) for thermotropic [5] and amphiphilic [6] liquid crystals, and fluorescence recovery after photobleaching (FRAP) for lyotropic (colloidal) systems [7]. Only a few studies have been performed where dynamical phenomena are tracked at the scale of the single anisotropic particle $[8,9]$.

In this work, the model system of aqueous dispersion of filamentous virus $f d$ particles, which exhibit a highly monodisperse length and width distribution and the ability to be visualised individually by fluorescence microscopy, has been used to explore 


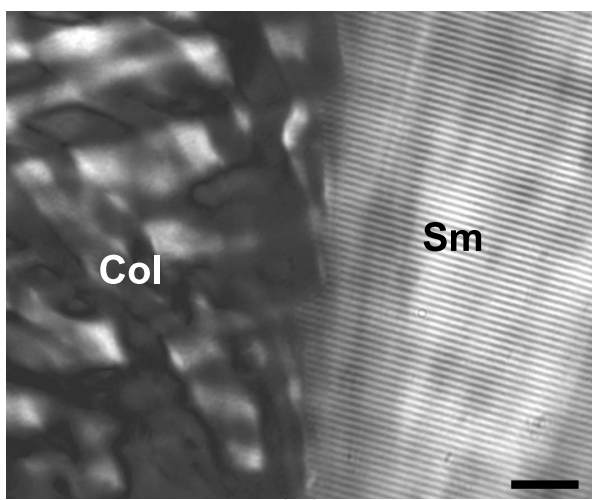

(a)

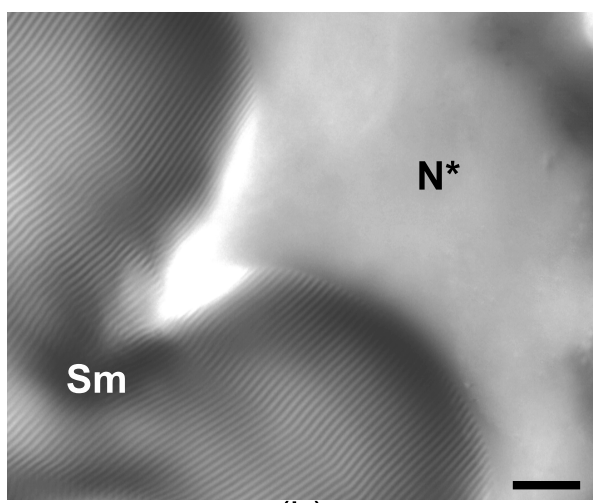

(b)

Figure 1. Phase coexistence of the different mesophases observed in aqueous suspensions of $f d$ rod-like viruses by differential interference contrast microscopy. Coexistence of the (a) columnar (left) and smectic (right) phases and of the (b) smectic (left) and chiral nematic (right) phases. The scale bars indicate $10 \mu \mathrm{m}$ in both cases.

the time-dependent phenomena in the smectic phase. In this lamellar mesophase, the particle density is quasi periodic in one dimension parallel to the long axis of the rods, while the interparticle correlations perpendicular to this axis are shortranged (fluid-like order). In the smectic phase of $f d$ virus suspensions, we investigate experimentally the process of interlayer diffusion or permeation, first predicted by Helfrich [10], corresponding to the jump along the long axes (or director) of single rod-like particles between adjacent smectic layers [9].

Here we first show that $f d$ dispersions undergo a first order nematic-smectic and smectic-columnar phase transition, using differential interference contrast microscopy. X-ray scattering is used to confirm that within the smectic layers rods show a Lorentzian radial distribution, typical for a liquid like ordering, but also for a glass. Having established the structural characteristics of the smectic phase, fluorescence video microscopy is employed to study self-diffusion in this lamellar mesophase. Although particles can supposedly diffuse freely within each liquid-like layer (with diffusion coefficient $D_{\perp}$ ) but must overcome a free energy barrier to jump between adjacent layers (with diffusion coefficient $D_{\|}$), surprisingly, the diffusion through the smectic layers is shown here to be much faster than the diffusion within each liquid-like layer, i.e. $D_{\|} / D_{\perp} \gg 1$. This behaviour will be analyzed in terms of a recently developed time dependent density functional functional theory [11].

\section{Experimental section}

\subsection{Materials and methods}

The system of rods used in this work consists of filamentous bacteriophages $f d$, which are semi-rigid polyelectrolytes with a contour length $L=0.88 \mu \mathrm{m}$, a diameter $d=66 \AA$, a persistence length of $2.2 \mu \mathrm{m}$, and a molecular weight of $\mathrm{M}_{W}=1.64 \times 10^{7} \mathrm{~g} / \mathrm{mol} . \mathrm{fd}$ was 

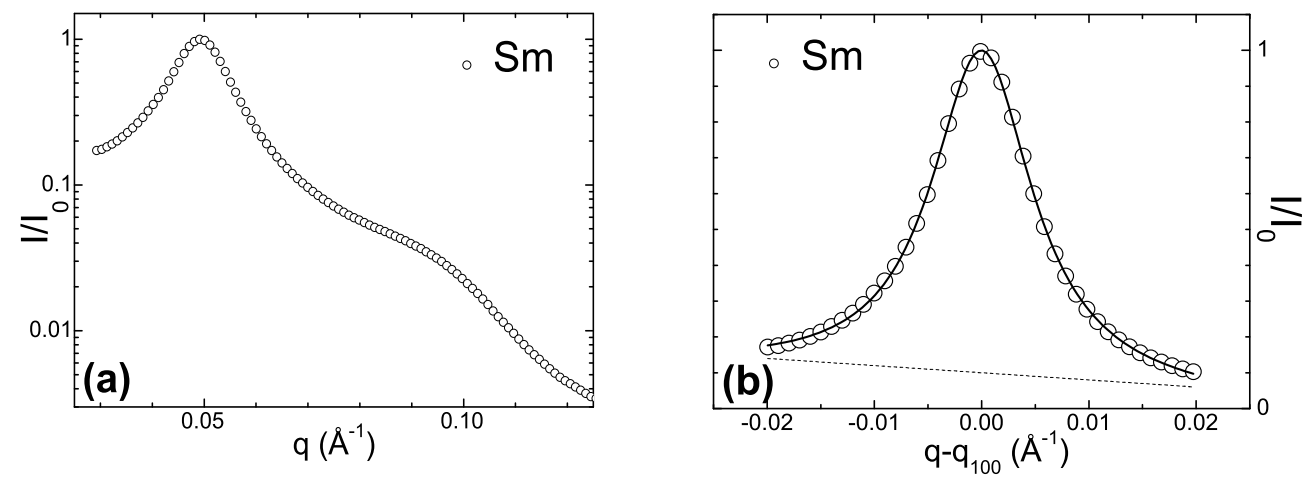

Figure 2. (a): Average radial intensity as a function of the scattering wave vector, probing the inter-rod structure within the smectic layer. (b): Lorentzian fit (solid line) of the first order Bragg reflection (open symbols) which are not resolution limited. The dashed line shows the subtracted linear background.

grown using the XL1-Blue strain of E. Coli as the host bacteria and purified following standard biological protocols [3]. In this study, the ionic strength has been fixed at $\mathrm{I}=20 \mathrm{mM}$ by a dialysis of $f d$ suspensions against a TRIS-HCl-NaCl buffer at $\mathrm{pH}=$ 8.2. At this $\mathrm{pH}$, the $f d$ charge density is about $10 \mathrm{e} / \mathrm{nm}$. The virus concentrations were measured using spectrophotometry with an absorption coefficient of $3.84 \mathrm{~cm}^{2} / \mathrm{mg}$ at $269 \mathrm{~nm}$. Video fluorescence microscopy has been used to monitor the dynamics of individual labeled colloidal rods in the background of a smectic mesophase formed by identical but unlabeled rods, where about one $f d$ rod out of $10^{4}$ has been labeled with the dye Alexa-488 (Invitrogen). The colloidal scale of the $f d$ bacteriophage enables the imaging of individual rods by fluorescence microscopy, as well as smectic layers by differential interference contrast (DIC) microscopy [3].

\subsection{Phase diagram and structural investigations}

Suspensions of $f d$ rods in aqueous solution form several lyotropic liquid crystalline phases with increasing particle concentration, ranging from the chiral nematic $\left(\mathrm{N}^{*}\right)[12]$ via the smectic $(\mathrm{Sm})[9,13]$ to columnar $(\mathrm{Col})$ and crystalline phases [14]. The existence of a smectic phase in suspensions of hard rods is an evidence of the high monodispersity in the particle length and therefore of the model system character of such filamentous viruses [15]. A conceptually appealing intuitive explanation for the appearance of the smectic phase was given by Wen and Meyer [16], and it goes as follows. In the uniaxial nematic phase, neighbouring rods overlap each other by random amounts along their principal direction. This creates volumes at the end of every rod, which are accessable only to that rod but not to any other rod. In the smectic phase, with rods distributed in layers, the random overlapping of rods along their length is avoided, so these excluded volumes disappear thereby increasing the free volume of the system. Hence, though positional entropy is lost at the transition to the smectic phase, freely available volume is gained and therefore the overall configurational entropy. 
At $\mathrm{I}=20 \mathrm{mM}$, the typical virus concentration for the smectic phase to occur is $115 \mathrm{mg} / \mathrm{ml}$, which corresponds to a volume fraction $\phi=0.13$. The volume fraction has been calculated with the bare virus diameter, and not with an effective diameter taking into account the electrostatic interactions between rods. Figure 1 presents the phase coexistence of the smectic phase with the chiral nematic and columnar mesophases, respectively. Both Col-Sm and $\mathrm{Sm}-\mathrm{N}^{*}$ phase transitions are first order, and they are fully reversible by dilution or concentration of the sample. Note that a sufficiently pronounced particle length polydispersity has been shown to rule out the smectic phase $[5,6]$ and that rod flexibility also destabilises the smectic organisation [17]. Another consequence of the virus flexibility is that the smectic layer spacing is very close to the particle length [18, 13].

In order to study the nature of the positional order within the smectic layers, small angle x-ray scattering (SAXS) has been performed at the ESRF-ID02 beamline (Grenoble, France). Figure 2(a) presents the average radial intensity in the wavevector range suitable for probing the interaxial organisation of the rods. The position of the Bragg peak is $q_{100}=0.0492 \AA^{-1}$, which corresponds to a distance between rods of $d_{\text {inter }}=4 \pi / \sqrt{ } 3 q_{100}=147 \AA$. In a conventional liquid the positional correlations decay exponentially with distance, giving a Lorentzian scattering profile of the Bragg reflections. A line shape analysis of the first order Bragg peak has been performed as shown in figure 2(b): a Lorentzian distribution almost perfectly fits the data. A positional correlation length of $\xi=2 \pi / \mathrm{FWHM}=540 \AA$ is found, which corresponds to an inter particle correlation extending up to about four neighbours. This demonstrates that the structure of the order is liquid-like in the layers of the smectic phase.

\subsection{Self-diffusion of single particles}

Figure 3(a) shows an example of images of a single region where both DIC and fluorescence techniques are combined: some rods jump between two layers while others remain within a given layer. The trajectory of one of the rods is plotted in figure 3(b) in the direction parallel $(\mathrm{z})$ and perpendicular $(\mathrm{x})$ to the director. The main result of our measurements is the following: diffusion between the smectic layers takes place in quasi-quantised steps of one rod length, and the diffusion within the smectic player is extremely slow.

The "hopping-type" diffusion is the consequence of the underlying ordering potential of the smectic phase and the vacancies available in adjacent layers. It shows that the mass transport between the layers is a discontinuous process, as evidenced by the self Van Hove function G(z,t) in figure 4(a) [19], which is defined as the probability density for a displacement $z$ during a time interval $t$ :

$$
G(z, t)=\frac{1}{N}\left\langle\sum_{i=1}^{N} \delta\left[z+z_{i}(0)-z_{i}(t)\right]\right\rangle .
$$

For an uniform fluid of Brownian particles, a smooth gaussian distribution that smears out over time is expected for the self Van Hove function. In the smectic phase, 

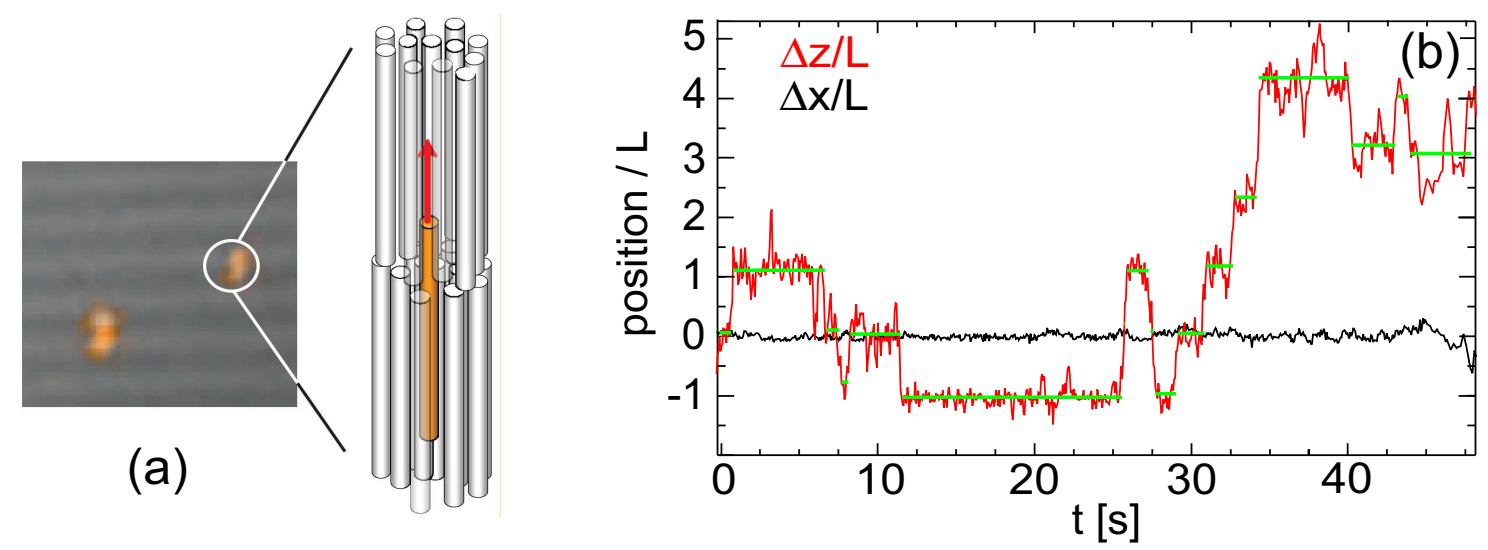

Figure 3. (a) Overlay of differential interference contrast and fluorescence images, showing the smectic layers and two fluorescently labeled particles, and the schematic representation of the jump of rod-like particle between adjacent smectic layers. The layer spacing is $L \simeq 0.9 \mu \mathrm{m}$. (b) Displacement of a given particle in the direction parallel (red line) and perpendicular (black line) to the normal of the smectic layers. The green lines indicate the residence time, i.e. the time for which one particle stays in a given layer.

however, $\mathrm{G}(\mathrm{z}, \mathrm{t})$ shows distinct peaks exactly at integer multiples of the particle length (and therefore of the layer thickness), as also inferred from visual inspection of the rod trajectories (Figure 3).

\subsection{Mean square displacement}

The overall mean square displacement (MSD) of rods parallel and perpendicular to the director of the smectic and nematic phases is plotted in figure 4(b). Here parallel MSD is scaled by the length of the $\operatorname{rod} L$, while the time is scaled by the time it takes to diffuse one rod length in the nematic phase, i.e. $\tau_{L}=L^{2} / D_{\|}^{n e m}$. Similarly, the perpendicular MSD is scaled by the rod diameter $d$, while the time is scaled by the time it takes to diffuse one rod thickness in the nematic phase, i.e. $\tau_{d}=d^{2} / D_{\perp}^{n e m}$. The time evolution of the MSD given by $\left\langle\Delta r^{2}(t)\right\rangle \sim t^{\gamma}$ provides the diffusion exponent $\gamma: \gamma<1$ is characteristic of a sub diffusive behavior, while $\gamma>1$ is referred to as super diffusion. The parallel motion is close to be diffusive in the (chiral) nematic phase $(\gamma=0.95)$ close to the $\mathrm{N}^{*}$-Sm phase transition over the whole studied time range, i.e. over several rod lengths. However, the parallel motion in the smectic phase is significantly sub diffusive for $t<\tau_{L}: \gamma=0.77$, while it is diffusive for $t>\tau_{L}(\gamma=0.96)$. The perpendicular motion is in both cases strongly sub diffusive. In the nematic phase $\gamma=0.68$, while in the smectic phase again two regimes can be distinguished: $\gamma=0.38$ for $t<1000 \tau_{d}$ and $\gamma=0.57$ for $t>1000 \tau_{d}$. 

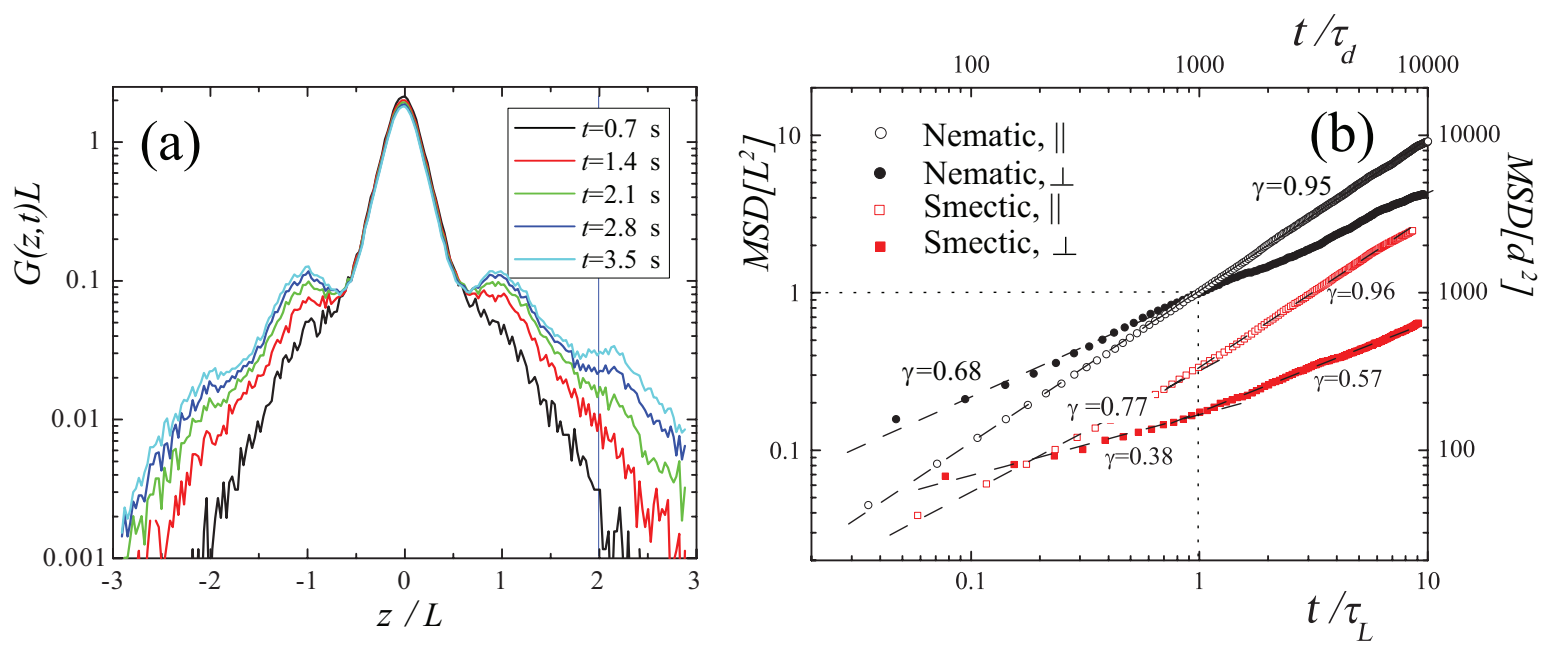

Figure 4. (a) Evolution of the self Van Hove function at different times. The functions are normalised to one, the $\mathrm{z}$-axis is scaled by the smectic layer thickness $L$. (b) Log-log representation of the scaled mean square displacement (MSD) parallel and perpendicular to the director in the nematic and smectic phases (see legend) vs. the scaled time. The dotted lines indicate the time the rods diffuse one rod length. The dashed lines represent the numerical fits by a power law.

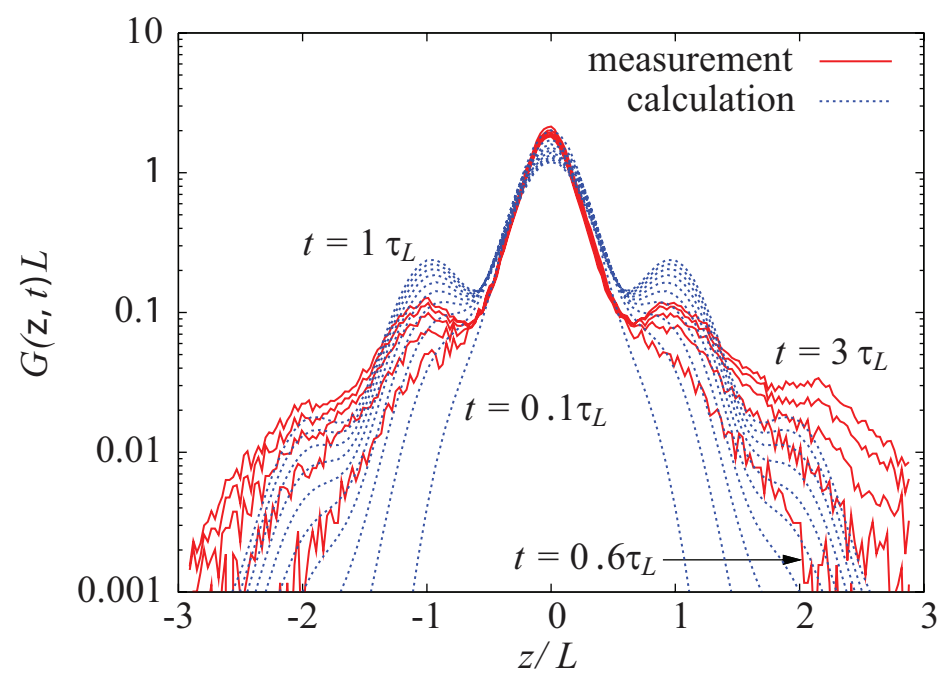

Figure 5. The self Van Hove correlation function $G(z, t)$ obtained within the dynamical density functional calculation based on a highly idealised second virial model of perfectly aligned, perfectly rigid hard rods indicated by the blue dotted lines exhibits the qualitative features of the measurement (red solid lines). Here $L$ is the smectic layer spacing and $\tau_{L}$ is a parallel diffusion time. The quantitative differences can be attributed to an overestimation of the compressibility and the neglect of the particle bending flexibility within the theoretical model. 


\section{Theoretical section}

In order to theoretically study the diffusion in uniform and non-uniform complex fluids, a general method was put forward allowing for the straightforward calculation of Van Hove correlation functions within dynamical density functional theory [11]. Because the $f d$ virus filaments can be considered as long, thin rods of high stiffness (see Subsection 2.1) that are strongly aligned in the nematic and smectic phases, one can in a first approximation neglect the orientational degrees of freedom [20] and model a liquid crystalline $f d$ virus dispersion as a fluid of aligned hard rods of an effective length and diameter. Within the dynamical density functional theory we next invoke the second virial approximation [21], which is not quite exact at the densities where the smectic phase is stable but contains the relevant physics at hand, and numerically solve the relevant kinetic equations that link the self diffusion of a test particle to the collective diffusion of all the other particles in the system.

In figure 5 we compare the self Van Hove correlation function $G(z, t)$ obtained from our model calculations with the measurements displayed in figure 4 . Here $L$ denotes the smectic layer spacing and $\tau_{L}$ is the parallel diffusion time $\tau_{L}=L^{2} / D_{\|}^{\text {short }}$, where $D_{\|}^{\text {short }}$ is the short time parallel diffusion. The model parameters for the smectic state in the calculation were chosen such that the smectic ordering potential barriers correspond to those determined in the experiment from a Boltzmann weighing of the density profiles.

Figure 5 shows that even a calculation at the level of the second virial approximation can account for the qualitative features of the non-trivial, hopping-type diffusive behaviour of rod-like particles along the director from one to the next smectic layer. The quantitative differences between the measurements and the theory can be understood by realising that the second virial approximation overestimates the compressibility of the fluid. Indeed, a rod in the model fluid can, compared to real $f d$ virus, more easily squeeze into a neighbouring layer, which increases the decay rate in the central region around $z=0$ and the growth rate of the first side peaks around $z= \pm L$ in figure 5 . The height of the peaks around $z= \pm 2 L$ is associated with the cooperative movement of a rod from the central layer via a void in the first layer to the second layer, which is more pronounced in the experience than in the calculation.

\section{Discussion}

Anomalous sub diffusive behaviour has often been observed in systems where diffusion involves an initial waiting time, e.g. following the release of a test particle from a temporary cage caused by the presence of other particles [22]. It stands to reason that this "cage escape" might be at the root of the observed sub diffusive behaviour for both parallel and perpendicular diffusion observed in the smectic phase. For parallel diffusion of rods the cage is actually formed by the free energy barrier imposed by the smectic layers, superimposed on which is the effective barrier from the enhanced number of particles that cage the central (test) particle [11]. Indeed both in the experiments and 
in the calculations (see also figure 3(a) in [11]) the parallel behaviour is sub diffusive for $t<\tau_{L}$, related to the crossover between short-time and long-time diffusive behaviour.

The anisotropy in the diffusivities, $D_{\|} / D_{\perp}$, which is about 20 in the nematic phase [8], increases in the smectic phase within the measured time range as a result of the pronounced sub diffusivity of the perpendicular motion (as is evidenced by the decrease of $\gamma$ ). Since this sub diffusive behavior lasts for the whole studied time range, i.e. thousands of rod diameters, it seems that the rods in the layers are glass-like rather then liquid like. This observation is apparently opposite to the trend found for thermotropic liquid crystals, where usually $D_{\|} / D_{\perp}$ decreases due to an Arrhenius form of the diffusion constants $[4,5]$. Also note that preliminary results by Dogic on the self diffusion of $f d$ virus particles in single lamellar membranes indicate that without neighboring layers perpendicular diffusion is much faster [23].

The cause of the experimentally observed perpendicular sub diffusive behaviour is not clear a priori. The theoretical calculations show diffusive long-time behaviour in the perpendicular direction; it should be realised, however, that both flexibility and orientational degrees of freedom are not taken into account in the theory, both of which will result in significant excluded volume effects. Thus, the dominant mode of perpendicular diffusion could be a reptation-like parallel motion of the rod along the long axis to escape its locally excluded volume, similar in nature to what is observed for polymers in the dense melt for which typically $\gamma=0.5$ [24]. Including these effects could also shine a light on the discrepancy between the envelope of the measured and calculated Van Hove functions plotted in figure 5, since the experimentally observed $\sim z^{-1}$ behaviour could be related to the relaxation of voids once a rod has jumped between two adjacent layers.

\section{Summary}

We have shown by means of real-space video fluorescence microscopy that the diffusive transport of particles between the layers of a smectic lyotropic colloidal liquid crystal is a discontinuous process that occurs in steps of one layer spacing. Our dynamical density functional theoretical approach, which is found to describe the underlying dynamics qualitatively, points out the importance of the existence of free energy barriers between the smectic layers. This gives rise to a kinetics where particles hop from one layer to the other with a time scale which is dictated by the height of the barriers. At shorter time scales, the particles remain trapped in the smectic layers and perform a diffusive "bobbing" motion about the local minimum of the self consistent molecular field.

\section{Acknowledgments}

We thank Marjolein Dijkstra for suggesting the application of DDFT to smectics of rods. This project was supported by the European network of excellence SoftComp and Transregio Sonderforschungsbereich TR6018 "Physics of Colloidal Dispersions in 
External Fields". This work is part of the research programme of the 'Stichting voor Fundamenteel Onderzoek der Materie (FOM)', which is financially supported by the 'Nederlandse Organisatie voor Wetenschappelijk Onderzoek (NWO)'.

\section{References}

[1] Frenkel D, Lekkerkerker H N W and Stroobants A 1988 Nature 332, 822; Vroege G J and Lekkerkerker H N W 1992 Rep. Prog. Phys. 55, 1241

[2] Wen X, Meyer R B and Caspar D L D 1989 Phys. Rev. Lett. 63, 2760; Davidson P and Gabriel J C P 2005 Curr. Opin. Colloid Interface Sci. 9, 377; Dogic Z and Fraden S 2006 Curr. Opin. Colloid Interface Sci. 11, 47

[3] Dogic Z and Fraden S 2006 in Soft Matter, edited by Gompper G and Schick M (Wiley-VCH, Weinheim), Vol. 2

[4] Hess S, Frenkel D and Allen M P 1991 Molec. Phys. 74, 765; Löwen H 1999 Phys. Rev. E 59, 1989; Selinger R L B 2002 Phys. Rev. E 65, 051702; Bates M A and Luckhurst G R $2004 \mathrm{~J}$. Chem. Phys. 120, 394; Cifelli M, Cinacchi G and De Gaetani L 2006 J. Chem. Phys. 125, 164912

[5] Krüger G J 1982 Phys. Rep. 82, 229; Dvinskikh S V, Furo I, Zimmermann H and Maliniak A 2002 Phys. Rev. E 65, 061701

[6] Blinc R, Easwaran K, Pirs J, Volfan M and Zupancic I 1970 Phys. Rev. Lett. 25, 1327; Gaemers S and Bax A $2001 \mathrm{~J}$. Am. Chem. Soc 123, 12343; Hubbard P L, McGrath K M and Callaghan P T 2005 Langmuir 21, 4340

[7] Bu Z, Russo P S, Tripton D L and Negulescu I I 1994 Macromolecules 27, 6871; van Bruggen M P B, Lekkerkerker H N W, Maret G and Dhont J K G 1998 Phys. Rev. E 58, 7668; Cush R C and Russo P S 2002 Macromolecules 35, 8659

[8] Lettinga M P, Barry E and Dogic Z 2005 Europhys. Lett. 71, 692

[9] Lettinga M P and Grelet E 2007 Phys. Rev. Lett. 99, 197802

[10] Helfrich W 1969 Phys. Rev. Lett. 23, 372

[11] Bier M, van Roij R, Dijkstra M and van der Schoot P 2008 Preprint

[12] Lapointe J and Marvin D A 1973 Mol. Cryst. Liq. Cryst. 19, 269; Dogic Z and Fraden S 2000 Langmuir 16, 7820; Grelet E and Fraden S 2003 Phys. Rev. Lett. 90, 198302; Tombolato F, Ferrarini A and Grelet E 2006 Phys. Rev. Lett. 96, 258302

[13] Dogic Z and Fraden S 1997 Phys. Rev. Lett. 782417

[14] Grelet E 2008 Phys. Rev. Lett. 100, 168301

[15] Bates M A and Frenkel D 1998 J. Chem. Phys. 109, 6193

[16] Wen X and Meyer R B 1987 Phys. Rev. Lett. 59, 1325

[17] Hentschke R and Herzfeld J 1991 Phys. Rev. A 44, 1148; Selinger J V and Bruinsma R F 1991 Phys. Rev. A 43, 2922; van der Schoot P 1996 J. Phys. II France 6, 1557

[18] van der Schoot P 1997 J. Phys. II France 6, 1557; Tkachenko A V 1996 Phys. Rev. Lett. 77, 4218

[19] Hansen J P and McDonald I R 1986 Theory of Simple Liquids (Academic Press, London)

[20] Odijk T 1986 Macromolecules 19, 2313

[21] Mulder B 1987 Phys. Rev. A 35, 3095

[22] Weeks E R and Weitz D A 2002 Phys. Rev. Lett. 89, 095704

[23] Dogic Z Private communication

[24] McLeish T C B 2002 Advances in Physics 51, 1379 